\title{
Relocated Colour Contrast Occurrence Matrix and adapted similarity measure for colour texture retrieval
}

\author{
H. Jebali ${ }^{1}$, N. Richard ${ }^{2}$, H. Chatoux ${ }^{2}$, and M. Naouai ${ }^{1}$ \\ 1 Faculty of Science of Tunis, University campus el Manar, Tunisia \\ jebalii.hela@gmail.com \\ ${ }^{2}$ University of Poitiers, XLIM UMR CNRS 6172, France \\ noel.richard@univ-poitiers.fr
}

\begin{abstract}
For metrological purposes, distance between texture images is crucial. This work study as a pair the couple texture feature/similarity measure. Starting from the Colour Contrast Occurrence Matrix $\left(\mathrm{C}_{2} \mathrm{O}\right)$ definition, we propose an adapted similarity measure improving texture retrieval. In a second step, we propose a modified version of the $\mathrm{C}_{2} \mathrm{O}$ definition including the texture's colour average inside a modified similarity measure. Performance in texture retrieval is assessed for four challenging datasets: Vistex, Stex, Outex-TC13 and KTH-TIPS2b databases facing to the recent results from the state-of-the-art. Results show the high efficiency of the proposed approach based on a simple pair feature/similarity measure facing to more complex approaches including Convolutional Neural Networks.
\end{abstract}

\section{Introduction}

Texture provides a semantic description to characterize the similarity between images. Although, different efficient texture descriptors have been developed in the literature. Cooccurrence matrix and Run-Length Matrix (RLM) [28] are good descriptors of texture content, but are not suitable for direct use with a distance or similarity measure. The Local Binary Pattern [21] attribute retains only a very reduced part of texture information about spatial-frequency composition. Yet, it is suitable for a distribution operation with matching metrics of similarities. Nevertheless the pair attribute and similarity measure is very efficient for texture classification or discrimination.

The transition to colour images is not straightforward with the vector aspect of the pair descriptor/measure of similarity. Marginal and/or crossed marginal (channel by channel) approaches can produce simplest descriptors but sliced the difficulty of the vector data management to the similarity measure function. It exists few vector expressions of texture features, among them the Colour Contrast Occurrence $\left(\mathrm{C}_{2} \mathrm{O}\right)$ matrix [22] proposes a vector descriptor producing a distribution of colour differences present in an image or region of interest. In previous work, we shown the metrological potential of the $\mathrm{C}_{2} \mathrm{O}$ [22], but in the 
same time the performances in texture classification were not as expected. In this work, we study the impact of the similarity metric on classification performance, and we propose an improved version of the $\mathrm{C}_{2} \mathrm{O}$ definition more adapt to texture image retrieval in databases.

In the following, we first describe the Colour Contrast Occurrence $\left(\mathrm{C}_{2} \mathrm{O}\right)$ construction (sec. 2). Then, we develop the question of similarity measurement between trichromatic distributions (sec. 3). Considering the invariance of the $\mathrm{C}_{2} \mathrm{O}$ to the average lightning change, we propose the Relocated Colour Contrast Occurrence matrix $\left(\mathrm{RC}_{2} \mathrm{O}\right)$ and an adapted expression of the similarity (sec. 3.4). In a first experiment, similarity measure performances are studied from some images of classic image databases (KTH, Stex). Then we compare performances of the $\mathrm{RC}_{2} \mathrm{O}$ using the proposed similarity metrics with a recent of state of the art approaches for Vistex, Stex, Outex-TC13 and KTH-TIPS2b databases (sec. $4.2)$.

\section{Color contrast occurrence matrix}

The $\mathrm{C}_{2} \mathrm{O}$ texture feature is inspired from the first Julesz conjecture ${ }^{3}$ [12]. The initial idea was to define a compact probability density function adapted to direct distance/similarity measurements between features.

The colour contrast occurrence matrix proposed in [17] expresses the probability $\Lambda\left(\overrightarrow{c_{i} c_{j}}\right)$ to find a specific local colour difference $\overrightarrow{c_{i} c_{j}}=\vec{\chi}$. This probability is assessed between two pixels separated by a vector defined by a spatial distance $d$ and an orientation $\theta$. The colour difference is defined in spherical coordinates by a perceptual color difference measurement $\Delta E_{\vec{\chi}}$ established in CIELAB by two angles $(\alpha, \beta)$.

3 The first Julesz conjecture states that preattentive discrimination of textures is possible only for textures that differ on second-order correlation statistics.
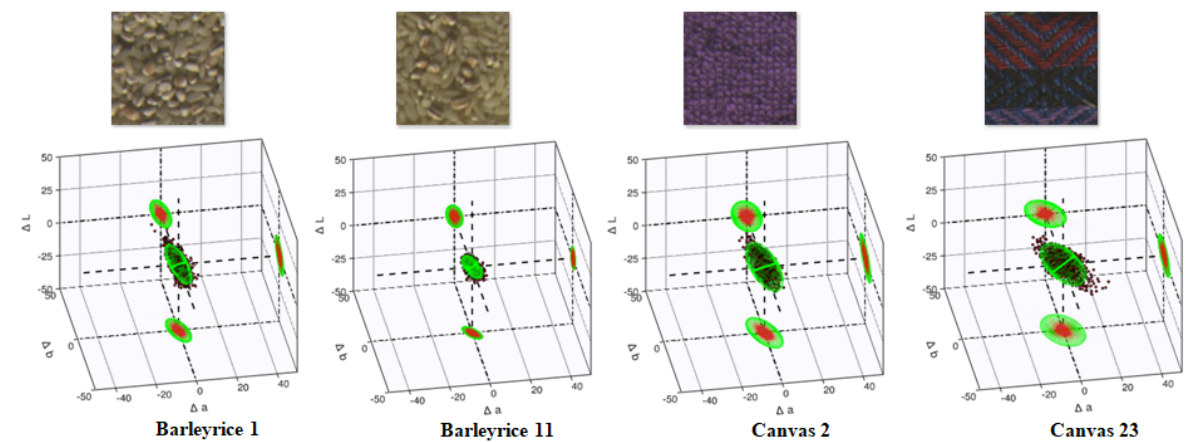

Fig. 1. Some color textures from Outex with their $C_{2} O$ distributions $(d=1, \theta=0)$ 


$$
\begin{aligned}
\overrightarrow{\Lambda\left(c_{i}, c_{j}\right)} & : \operatorname{prob}\left(\overrightarrow{\Lambda\left(c_{i}, c_{j}\right)}=\overrightarrow{\Lambda_{\chi}}\right) \\
& \text { With }\left\|\overrightarrow{\Lambda\left(c_{i}, c_{j}\right)}\right\|=\Delta E_{\chi} \\
& \text { and }\left\llcorner\left(\overrightarrow{O a}, \overrightarrow{c_{i} c_{j}}\right)=(\alpha, \beta)\right.
\end{aligned}
$$

Concretely, the $\mathrm{C}_{2} \mathrm{O}$ matrix is a probability density function expressed in a $\left(\Delta_{L}, \Delta_{a}, \Delta_{b}\right)$ space. When texture is stationary, the $C_{2} O$ matrix is centred at the origin in a dense distribution (examples in Figure 1).

\section{Similarity measure between $\mathrm{C}_{2} \mathrm{O}$ matrix}

\subsection{Indirect measurement}

The main objective of the $C 2 O$ construction was to obtain a compact 3-dimensional probability distribution functions associated to an adapted distance or similarity measure. The initial construction in [17] was associated to a quantification using a spiral path in order to obtain a texture signature $\operatorname{Sig}_{\mathrm{C}_{2} \mathrm{O}}(I)$ (Equation 2 and Figure 2). Then the distance between two textures is obtained using a $L_{2}$ norm between texture signatures.

$$
\begin{aligned}
\operatorname{Sig}_{C_{2} O}(I)= & \Delta_{i} \alpha_{j} \beta_{k} \\
= & \operatorname{prob}\left(\Delta_{i} \leq\left\|\overrightarrow{\Lambda\left(c_{i}, c_{j}\right)}\right\|<\Delta_{j}+\Delta E_{\text {step }}\right) \\
& \text { With } \quad \frac{\pi}{2 n_{\alpha}}(j) \leq \alpha<\frac{\pi}{2 n_{\alpha}}(j) \\
& \text { and } \quad 0 \leq \beta<\frac{\pi}{n_{\beta}}(k+1)
\end{aligned}
$$

where $\Delta E_{\text {step }}, n_{\alpha}$ and $n_{\beta}$ represent respectively the contrast norm, chromatic and lightning steps.
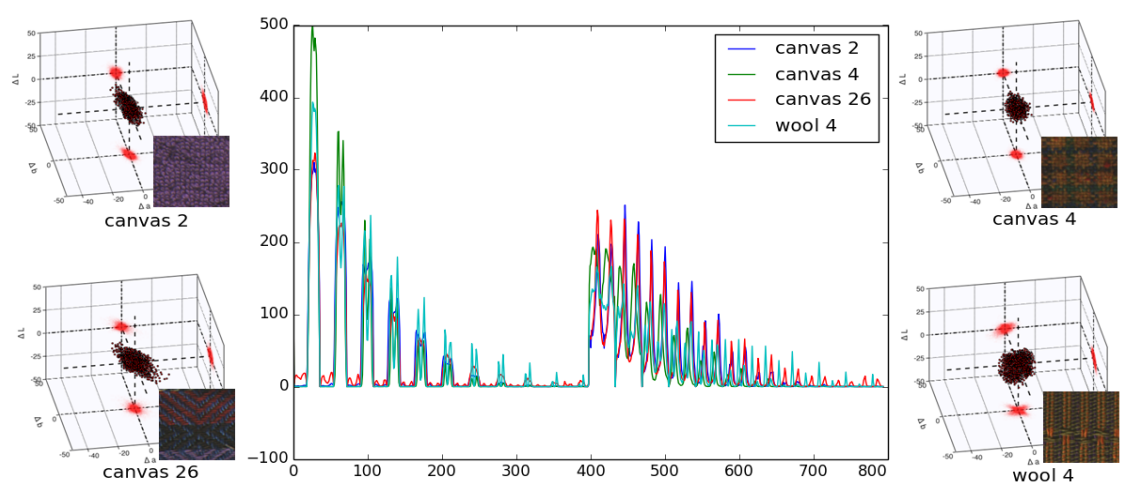

Fig. 2. $\mathrm{C}_{2} \mathrm{O}$ signature proposed in [17] for some Outex textures. The signature is not compact and present periodic patterns due to the distribution's quantification using a spiral path from the distribution center (Equation 2). 


\subsection{Measurement using Kullback Leibler divergence}

Considering $\mathrm{C}_{2} \mathrm{O}$ matrix as a probability density function, $f$-divergence are well adapted to assess the similarity between them. Inside this group, the KullbackLeibler divergence is the most used. In [8], the authors analysed and compared several similarity functions between colour distributions. In this work, they shown the Kullback-Leibler divergence takes into account all colour changes between the two considered distributions, whatever their magnitude. This divergence is also used for texture discrimination as in [18] using features based on energy from multi-scale Gabor-filters.

The Kullback-Leibler measure of information $K L(P / Q)$ assess the quantity of information lost when $Q$ is used to estimate $P$ (Equation 3) [14].

$$
K L(P / Q)=\int_{-\infty}^{\infty} p(x) \ln \left(\frac{p(x)}{q(x)}\right) d x
$$

where $\mathrm{p}$ and $\mathrm{q}$ are the respective densities of $P$ and $Q$. In order to define a similarity measure, the Kullback-Leibler divergence is defined as the sum of the measure of information of $P$ relative to $Q$ and the measure of information of $Q$ relative to $P$.

$$
\operatorname{div}_{K L}(P, Q)=K L(P / Q)+K L(Q / P)
$$

The direct application of the Kullback-Leibler divergence is costly. In order to solve this problem, Wang and Goldberger proposed methods accelerating this process but loosing in precision [10,27].

\subsection{Estimation of the KL-divergence}

Due to the difficulty and cost of a direct processing, adaptations of the KullbackLeibler measure of information were proposed for different types of distributions. For example, Mathiasan [18] proposed a dedicated estimation for Gamma distribution. Obviously, the most used case assumes that the probability density function can be assessed as a multivariate normal distribution. In this case, the KL measure of information is defined according to the average location $\mu$ and variance-covariance matrix $\Sigma$ of each distribution (Equation 5).

$$
K L(P / Q)=\frac{1}{2}\left(\log \left(\frac{\left|\Sigma_{Q}\right|}{\left|\Sigma_{P}\right|}\right)+\operatorname{tr}\left(\Sigma_{Q}^{-1} \Sigma_{P}\right)+\left(\mu_{Q}-\mu_{P}\right)^{t} \Sigma_{Q}^{-1}\left(\mu_{Q}-\mu_{P}\right)-3\right)
$$

The observations of different textures' $\mathrm{C}_{2} O$ distributions allow us to approximate them as multivariate normal distributions. Technically, the distribution can be approximated by a 3-dimensional ellipsoid in the $C_{2} O$ feature space $(\Delta L, \Delta a, \Delta b)$. The quality of this approximation can be observed in Figures 1,4 and 5 thanks to the drawn ellipsoids in green. The ellipsoid surface is plotted as being at a Mahalanobis distance of 3 units from the distribution average, hence $99.7 \%$ of the samples must be considered as being inside this ellipsoid. 


\subsection{Relocated Colour Contrast Occurrence}

The $\mathrm{C}_{2} \mathrm{O}$ matrix describes the internal colour variations of texture. When the texture is stationary, the $\mathrm{C}_{2} \mathrm{O}$ is a distribution centred at the origin of the representation space. For application in Quality Control, all textures belonging to the same category have a similar colour average. But in order to assess the performance of the pair feature/similarity measure, we search to adapt the purpose to texture classification from image database. In this case, the colour average of each image is different. As shown in a lot of studies on texture discrimination, the colour average can be important for the texture retrieval or classification [25].

We define the Relocated Colour Contrast Occurrence Matrix $\left(\mathrm{RC}_{2} \mathrm{O}\right)$ as being the distribution of the $\mathrm{C}_{2} \mathrm{O}$ relocated at the image colour disribution's average in the $C I E L A B$ colour space. Then the similarity measurement between two textures is assessed with the similarity between the two $\mathrm{C}_{2} \mathrm{O}$ distributions taking into account their colour average difference.

As the colour average and the $\mathrm{C}_{2} \mathrm{O}$ distribution are not calculated in parallel, we propose an easy way to process the similarity between the $\mathrm{RC}_{2} \mathrm{O}$ of two textures (Equation 6). The new measure of information, for the $R C_{2} O, K L c\left(S_{1}, S_{2}\right)$ is estimated from the estimation of the KL measure of information (Equation 5).

$$
\begin{aligned}
K L c\left(S_{1}, S_{2}\right)= & \frac{1}{2}\left(\log \left(\frac{\left|\Sigma_{2}\right|}{\left|\Sigma_{1}\right|}\right)+\operatorname{tr}\left(\Sigma_{2}^{-1} \Sigma_{1}\right)\right. \\
& \left.+\left(\mu_{S_{1}}+\mu_{1}-\mu_{S_{2}}-\mu_{2}\right)^{t} \Sigma_{2}^{-1}\left(\mu_{S_{1}}+\mu_{1}-\mu_{S_{2}}-\mu_{2}\right)-3\right)
\end{aligned}
$$

Where $\mu_{i}$ and $\Sigma_{i}$ represent respectively the mean and the variance-covariance matrix of the $C_{2} O$ descriptor of the image $i$, and $\mu_{S_{i}}$ represents the average

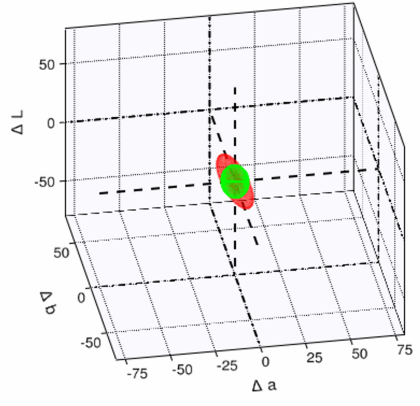

(a) $D_{K L}=2.276$

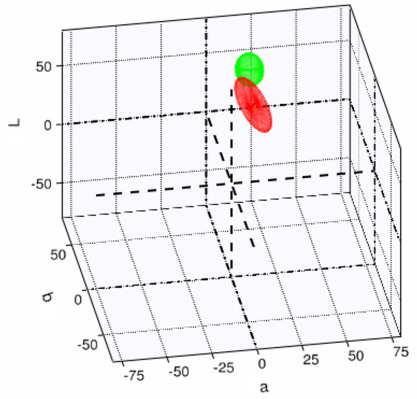

(b) $D_{K L_{c}}=184.052$

Fig. 3. Ellipsoid modelling of $\mathrm{C}_{2} \mathrm{O}$ and the proposed $R_{2} O$ distributions $(d=1, \theta=0)$ from two Vistex textures (Fabric and Flowers presented in Figure 4). As observed the $\mathrm{RC}_{2} \mathrm{O}$ distribution will induces a bigger difference between the two textures. 
coordinate of the image $i$ in the CIELAB space. The similarity measure between two image thanks to the Relocated Colour Contrast Occurrence Matrix is defined by the sum of the two KL measures of informations:

$$
d_{K L c}=K L c\left(S_{1}, S_{2}\right)+K L c\left(S_{2}, S_{1}\right)
$$

Figure 3 presents the similarity assessed by the initial writing form using the $\mathrm{C}_{2} \mathrm{O}$ distribution (Figures 3(a) and 4(a)) or using the Relocated Colour Contrast Occurrence Matrix (Figures 3(b) and 4(b)). As observed, the second solution induces the biggest measure of difference than the first one, only based on the differences of the local colour variations.

\section{Experiments and Discussion}

\subsection{Dataset and Results}

For experimentation, we use four challenging databases: Vistex, Stex, OutexTC13 and KTH-TIPS2b.

The classification is based on a nearest neighbour approach. The image is affected to the same class as its closest image from the learning set. So, each image category is defined by the union of the images from the learning set. This construction allows to take into account the lack of stationarity of some images coming from the natural world (vegetation typically).

The Outex [2] database is a large collection of 68 classes. Following Arvis's process [5] for the classification, each colour image was divided into 20 subimages of $128 \times 128$ pixels so the total of 1360 images. we take $50 \%$ of them for the training and the remaining $50 \%$ for validation.

The Figure 1 shows some Outex textures with their $\mathrm{C}_{2} \mathrm{O}$ distribution. Visually, we can notice the great similarity between the two classes Barleyrice 1 and Barleyrice 11. This similarity is validated firstly by their $C_{2} O$ distribution and then by the estimated distance $D_{K L}=2.052$ (see Table 1). On the other hand the Barleyrice 11 classe is far from the Canvas 2 texture in the feature space, that is expressed by the difference value $D_{K L}=90.435$.

Vistex test [3] is built of 54 colour images, whose initial size are $512 \times 512$ pixels, these images are acquired using uncontrolled conditions. As the Outex case,

Table 1. Inter and intra-class $D_{K L_{c}}$ distance for images of Outex database.

\begin{tabular}{|c|c|c|c|c|}
\hline Outex & Barleyrice & 1 Barleyrice & 11 Canvas & 2 Canvas 23 \\
\hline Barleyrice 1 & 0 & 2.052 & 50.634 & 39.782 \\
\hline Barleyrice 11 & 2.052 & $\mathbf{0}$ & 90.435 & 72.330 \\
\hline Canvas 2 & 50.634 & 90.435 & $\mathbf{0}$ & 17.233 \\
\hline Canvas 23 & 39.782 & 72.330 & 17.233 & $\mathbf{0}$ \\
\hline
\end{tabular}


each colour image was divided into 16 sub-images of $128 \times 128$ pixels, resulting 432 images. The Figure 4 presents some classes from Vistex with their $\mathrm{C}_{2} \mathrm{O}$ distributions while Table 2 shows the inter and intra-class $D_{K L_{c}}$ distance between them. The inter class distance is still zero while the intra-class distance varies according to the similarity between classes. The smallest distance is between the Fabric and the Food (5.025) which shows their big resemblance.
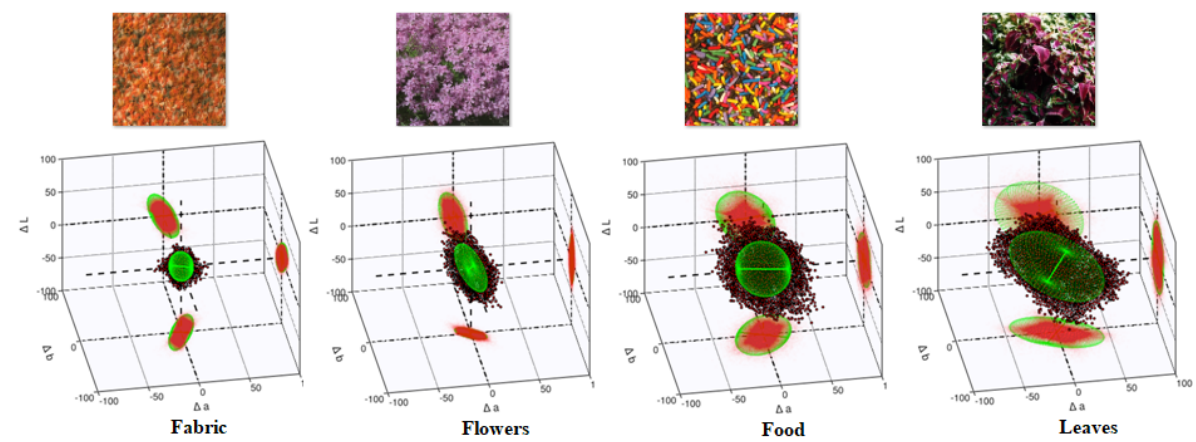

(a) $\mathrm{C}_{2} \mathrm{O}$ distributions
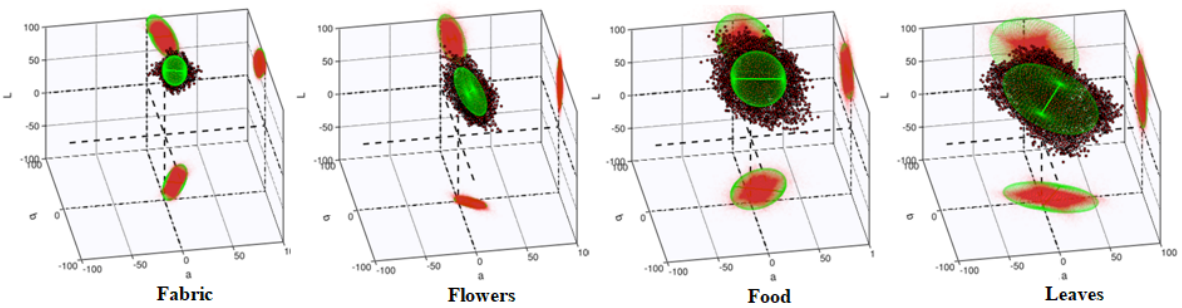

(b) Relocated $\mathrm{C}_{2} \mathrm{O}$ distributions

Fig. 4. Some color textures from Vistex with their: (a) $C_{2} O$ distributions $(d=1, \theta=0)$ and (b) Relocated $\mathrm{C}_{2} \mathrm{O}$ distributions.

Table 2. Inter and intra-class $D_{K L_{c}}$ distance for images of Vistex database.

\begin{tabular}{|c|c|c|c|c|}
\hline Vistex & Fabric & Flowers & Food & $\overline{\text { Leaves }}$ \\
\hline Fabric & 0 & 184.052 & 5.025 & 81.305 \\
\hline Flowers & 184.052 & $\mathbf{0}$ & 99.175 & 24.311 \\
\hline Food & 5.025 & 99.175 & 0 & 34.327 \\
\hline Leaves & 81.305 & 24.311 & 34.327 & $\mathbf{0}$ \\
\hline
\end{tabular}


The Stex database (Salzburg texture images) [1] is composed by 476 color texture images, whose acquisition conditions are not defined. Each colour image is split up into 16 non-overlapping $128 \times 128$ sub-images.

The KTH-TIPS2b [7] database includes 11 different texture classes with four samples of each. This database contains in total number of 4752 imges, which captured at nine different scales, four illumination conditions and three poses. The Figure 5 presents a Lettuce-leaf texture from the KTH-TIPS2b with different scale variations (scale 1 , scale 6 and scale 9 ). We can show the highest similarity measurement between all $\mathrm{C}_{2} \mathrm{O}$ distributions whose is almost stable for the two scales 6 and 9. The inter-class estimated distances are presented in Table 3 , all values are very weak which confirms the similarity between these textures. Therefore we can validate the $\mathrm{C}_{2} \mathrm{O}$ robustness to scale variation.
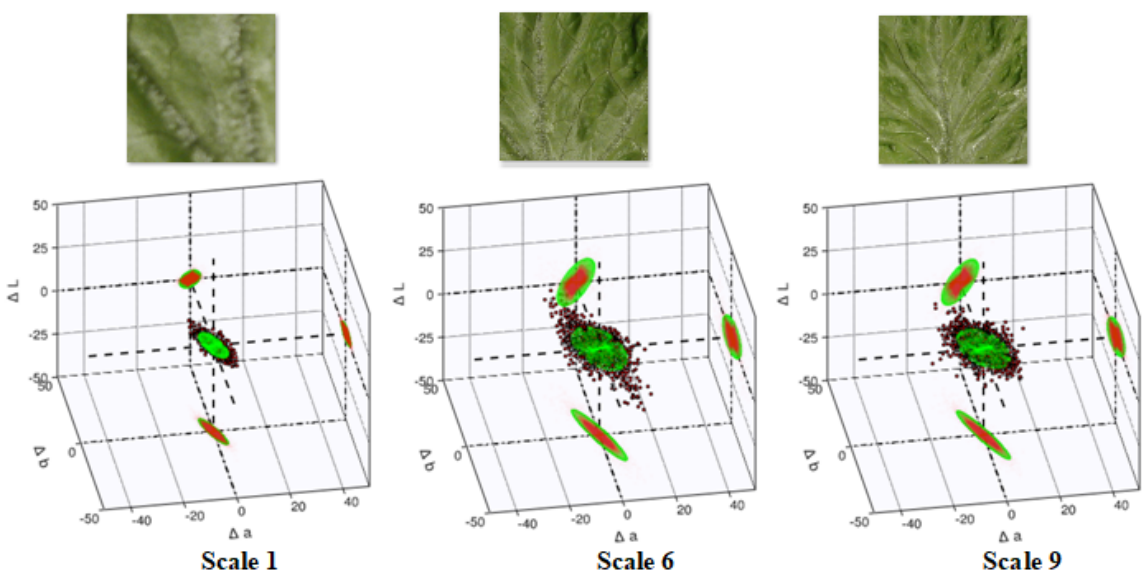

Fig. 5. Lettuce-leaf texture from KTH-TIPS2b database with their $\mathrm{C}_{2} \mathrm{O}$ distributions $(d=1, \theta=0)$ with different scale variation

Table 3. Inter-class $D_{K L_{c}}$ distance for Lettuce-leaf texture from KTH-TIPS2b database with different scale variation.

\begin{tabular}{|c|c|c|c|}
\hline Scale 1 & 0 & 5.201 & 4.323 \\
\hline Scale 6 & 5.201 & 0 & 0.696 \\
\hline Scale 9 & 4.323 & 0.696 & 0 \\
\hline
\end{tabular}




\subsection{Performance in Classification}

In this section, we present the obtained classification rates for our proposition based on the Relocated Colour Contrast Occurrence matrix $\mathrm{RC}_{2} \mathrm{O}_{D K L}$ in comparison to results recently published (Table 4).

Firstly, it can be observed that for three of the four datasets, the $R_{2} \mathrm{O}_{D K L}$ have the better classification rate : Vistex (100\%), Stex (87.24\%) and KTHTIPS2b (96.21\%). For the Outex database, the greatest classification rate is obtained by the $3 D-A S D H$ method (95.8\%) [24] and the third highest classification rate of $(92.4 \%)$ is achieved by our proposed approach after the LSTM network [6].

Secondly, we compare the gain of the proposed contribution to the initial $\mathrm{C}_{2} \mathrm{O}$ construction. For the Outex database, the content is essentially manufactured with reduced local colour intra-variations, but important colour variations between the textures. In this case, the good-classification score gain is around $12 \%$, in accordance with the information captured by the proposed $R_{2} O_{D K L}$. The other image databases have more images from the natural world, local variations (so the textured aspect) are more important than in the Outex case therefore the gain is reduced but always positive.

It is interesting to note that when the image database content becomes more complex with a bigger number of images from the natural world, the different approaches present smallest good-classification scores (case of Stex image database). In this case, an important part of images is not stationary.

Table 4. Comparison of the classification rates (\%) achieved on Vistex, Stex and Outex-TC13 and multi-scale KTH-TIPS2b databases. The proposed approach $\mathrm{RC}_{2} \mathrm{O}_{\mathrm{KL}}$ obtains good performances facing the initial $\mathrm{C}_{2} \mathrm{O}$ and more complex approaches using Convolutional Neural Networks.

\begin{tabular}{|c|c|c|c|c|}
\hline Methods & Vistex & Stex & $\begin{array}{c}\text { Outex } \\
\text { TC13 }\end{array}$ & $\begin{array}{c}\text { КТН } \\
\text { TIPS2b }\end{array}$ \\
\hline LED+ED [20] & 94.70 & 80.08 & 76.67 & - \\
\hline CLP $[23]$ & 97.70 & 83.90 & 82.10 & - \\
\hline LSTM network [6] & 99.09 & - & 94.7 & - \\
\hline 3D-ASDH [24] & - & - & 95.8 & 91.3 \\
\hline SMGD [16] & 97.5 & 77.6 & 89.7 & - \\
\hline GLACI $[15]$ & 88.42 & 41.91 & 87.67 & - \\
\hline CCMA [11] & 91.89 & 57.58 & 83.67 & - \\
\hline $\mathrm{BF}+\mathrm{CLBP}+\mathrm{S} / \mathrm{M}+$ ScatNet $[19]$ & - & - & - & 78.09 \\
\hline FC-CNN+FV-CNN $[26]$ & - & - & - & 83.3 \\
\hline STD [4] & 98.89 & - & 90.3 & - \\
\hline Compact DITC [13] & - & - & - & 69 \\
\hline $3 D$ connectivity $[9]$ & - & - & 84.26 & 83.54 \\
\hline $\mathbf{C}_{2} \mathbf{O}[17]$ & 99.3 & 76.65 & 82.64 & - \\
\hline $\mathrm{RC}_{2} \mathrm{O}_{\mathrm{DKL}}$ & 100 & 87.24 & 92.4 & 96.21 \\
\hline
\end{tabular}


To finish result analysis, we also note that the good performances of the pair $\mathrm{RC}_{2} \mathrm{O}$ feature/similarity measure are obtained compared to some approaches based on Deep-Learning solutions. These results show the interest of an accurate texture feature associated to an efficient similarity measurement function that obtain a good classification score on a basic classification scheme (1-NN).

\section{Conclusion}

In this paper, we proposed a new texture feature associated with an adapted similarity measure: the Relocated Colour Contrast Occurrence matrix associated to a Kullback-Leibler divergence. This texture feature characterizes the local colour difference processed in a perceptual colour space and take into account the colour average of the texture. The proposed construction is fully in accordance with the first Julesz conjecture. In order to consider the non-stationarity of some categories, each texture is considered as a collection of Relocated Colour Contrast Occurrence matrix.

The presented results show that the proposed texture feature is robust to different image changes and very efficient for texture image classification. The Relocated Colour Contrast Occurrence matrix presents the best classification score in three cases out of four, and is the third for the last one. These results will be extended to more image databases in order improve the ranking of this pair texture feature/similarity measure.

\section{References}

1. Salzburg texture image database stex, Department of Computer Sciences. http://www.wavelab.at/sources/STex

2. University of Oulu, Outex texture database. http://www.outex.oulu.fi

3. VisTex Vision Texture Database, Vision and Modeling Group, MIT Media Laboratory, 1995. http://vismod.media.mit.edu/vismod/imagery/VisionTexture

4. Alvarez, S., Vanrell, M.: Texton theory revisited: A bag-of-words approach to combine textons. Pattern Recogn 45(12), 4312 - 4325 (2012)

5. Arvis, V., Debain, C., Berducat, M., Benassi, A.: Generalization of the cooccurrence matrix for colour images: Application to colour texture segmentation. Image Anal Estereol 23, 63-72 (2004)

6. Byeon, W., Liwicki, M., Breuel, T.: Texture classification using 2d lstm networks. IEEE, 22nd Int. C. Pattern Recogn. (ICPR) (2014)

7. Caputo, B., Hayman, E., Fritz, M., Eklundh, J.: Classifying materials in the real world. Image Vision Comput 28, 150-163 (2010)

8. Chatoux, H., Richard, N., Lecellier, F., Fernandez-Maloigne, C.: Différence entre distributions couleur. ORASIS: 16ème journées francophones des jeunes chercheurs en vision par ordinateur (Jun 2017)

9. Florindo, J.B., Landini, G., Bruno, O.M.: Three-dimensional connectivity index for texture recognition. Pattern Recognition Letters 84, 239-244 (December 2016)

10. Goldberger, J., Gordon, S., Greenspan, H., al: An efficient image similarity measure based on approximations of kl-divergence between two gaussian mixtures. In ICCV $3,487-493(2003)$ 
11. Hauta-Kasari, M., Parkkinen, J., Jaaskelainen, T., Lenz, R.: Genaralized coocurrence matrix for multispectral texture analisis. 13th International Conference on Pattern Recognition I (August 1996)

12. Julesz, B.: Texture and visual perception. Scientific American 212, 38-48 (February 1965)

13. Khan, F.S., Anwer, R.M., van de Weijer, J., Felsberg, M., Laaksonen, J.: Compact color-texture description for texture classification. Pattern Recognition Letters 51, 16-22 (2015)

14. Kullback, S., Leibler, R.: On information and sufficiency. Ann. Math. Statist 22(1), 79-86 (1951)

15. Mäenpää, T., Pietikäinen, M.: Classification with color and texture: jointly or separately? Pattern Recognition 37, 1629-1640 (2004)

16. Maliani, A.D.E., Hassouni, M.E., Berthoumieu, Y., Aboutajdine, D.: Color texture classification method based on a statistical multi-model and geodesic distance. Journal of Visual Communication and Image Representation, Elsevier (2014)

17. Martnez, R., Richard, N., Fernandez, C.: Alternative to colour feature classification using colour contrast ocurrence matrix. Proc. SPIE 9534, Twelfth International Conference on Quality Control by Artificial Vision (30 April 2015)

18. Mathiassen, J.R., Skavhaug, A., Bo, K.: Texture similarity measure using kullbackleibler divergence between gamma distributions. Lecture Notes in Computer Science ECCV-LNCS 2352, 133-147 (2002)

19. Nguyen, V.L., Vu, N.S., Phan, H.H., Gosselin, P.H.: An integrated descriptor for texture classification. 23rd IEEE International Conference on Pattern Recognition (ICPR) (2016)

20. Pham, M.T., Mercier, G., Bombrun, L.: Color texture image retrieval based on local extrema features and riemannian distance. Journal of Imaging 3(4) (2017)

21. Porebski, A., Vandenbroucke, N., Hamad, D.: Lbp histogram selection for supervised color texture classification. ICIP pp. 3239-3243 (2013)

22. Richard, N., Ivanovici, M., Bony, A.: Toward a metrology for non-uniform surface using the complexity notion. In: 4th CIE Expert Symposium on Colour and Visual Appearance. pp. 40-50. Prague (Czech Republic) (Sept 2016)

23. Richard, N., Martnez, R., Fernandez, C.: Colour local pattern: a texture feature for colour images. Journal of the International Colour Association 16, 56-68 (2016)

24. Sandid, F., Douik, A.: Robust color texture descriptor for material recognition. Pattern Recognition Letters 80, 15-23 (2016)

25. S.Mangijao, S., Hemachandran, K.: Content-based image retrieval using color moment and gabor texture feature. In: IJCSI International Journal of Computer Scienc. vol. 9, pp. 299-309 (September 2012)

26. Song, Y., Li, Q., Feng, D., Zou, J.J., Ca, W.: Texture image classification with discriminative neural networks. Ann. Math. Statist 2(4), 367-377 (2016)

27. Wang, Q., Kulkarni, S., Verdú, S.: Divergence estimation for multidimensional densities via k-nearest-neighbor distances. IEEE Transactions on Information Theory (55) (2009)

28. Xiaoyan, S., Shao-Hui, C., Jiang, L., Frederic, M.: Automatic diagnosis for prostate cancer using run-length matrix method. Medical Imaging. Procceding of SPIE 7260 (2009) 